\title{
Aplicaciones de la teoría de juegos en el proceso de gestión estratégica
}

\section{Applications of game theory in the strategic management process}

Marcos España García

Universidad de Guayaquil, Ecuador

Freddy Correa Velasco

Universidad de Guayaquil, Ecuador

Autor para correspondencia: marcos.espanaga@ug.edu.ec, freddy.correave@ug.edu.ec Fecha de recepción: 14 de Mayo de 2017 - Fecha de aceptación: 15 de Septiembre de 2017

\section{Resumen}

El propósito de esta investigación es establecer las diferentes aplicaciones de la teoría de juegos en la administración estratégica en general; para lo cual se realiza una revisión bibliográfica que nos ayuda a establecer las diferentes maneras en que la teoría de juegos se ha aplicado en la administración estratégica de las organizaciones tanto públicas como privadas. Se establece que el actual nivel de competitividad de los mercados provocan guerras de precios en los que las empresas se ven involucradas y esta guerra de precios tiene como consecuencias pérdidas para todas las empresas involucradas, pero la reducción de aprecios es una estrategia que dependiendo del mercado y como se la aplique puede generar ganancias en algunos competidores.

Palabras Clave: teoría de juegos; gestión estratégica; estrategia; cooperación

\begin{abstract}
The purpose of this research is to establish the different applications of game theory in strategic management in general; For which a bibliographic review is done that helps us to establish the different ways in which game theory has been applied in the strategic administration of both public and private organizations. It is established that the current level of market competitiveness provokes price wars in which companies are involved and this price war has as a consequence losses for all the companies involved, but the reduction of appreciation is a strategy that depending on the market And how to apply it can generate profits in some competitors.
\end{abstract}

Key words: game theory; strategic management; strategy; cooperation 


\section{Introducción}

La teoría de juegos con las grandes bases matemáticas que la sostienen, ayuda a resolver muchos problemas que se presentan en las organizaciones ya sean públicas o privadas. El propósito de esta investigación es establecer las diferentes aplicaciones de la teoría de juegos en la administración estratégica en general; para lo cual se realiza una revisión bibliográfica que nos ayuda a establecer las diferentes maneras en que la teoría de juegos se ha aplicado en la administración estratégica de las organizaciones tanto públicas como privadas. La importancia de esta investigación radica en lo necesario que es para un administrador conocer esta herramienta y las situaciones en que puede ser aplicada, para la solución óptima de conflictos, ya que vivimos en un mundo competitivo en donde las empresas compiten por los recursos que en muchas ocasiones son limitados como el dinero, el personal calificado, el mercado, etc., y en esa competencia muchas veces existen dos o más competidores por lo que dichos conflictos caen dentro de la Teoría de Juegos, ya que, la teoría de los juegos puede usarse para predecir cómo se comportan las personas, siguiendo sus propios intereses en los conflictos (Madan, 2010). En un juego típico (un conflicto), los que toman las decisiones (los jugadores), con sus propios objetivos, tratan de superar a los demás al anticipar la decisión del otro; el juego se resuelve como consecuencia de las decisiones de los jugadores y la teoría de juegos analiza las estrategias que usan los jugadores para maximizar sus ganancias (Madan, 2010). Se tiene que los resultados pronosticados por la teoría de juegos a menudo difieren de los resultados sugeridos y los métodos de optimización que asumen que todas las partes están dispuestas a actuar hacia el mejor resultado en todo el sistema; pero, los comportamientos de las partes interesadas, que podrían estar dispuestos a contribuir a las mejoras y alcanzar una situación de ganar-ganar, a veces dan lugar a condiciones peores para todas las partes, porque la principal preocupación de los jugadores es maximizar su propio beneficio en el juego sabiendo que el resultado final es el producto de todas las decisiones tomadas (Madan, 2010).

La teoría de los juegos es esencialmente el estudio matemático de la competencia y la cooperación, ya que ilustra cómo las interacciones estratégicas entre los jugadores producen resultados globales con respecto a las preferencias de esos jugadores (Madan, 2010). Además, la teoría de juegos ofrece una simulación más realista del comportamiento basado en los intereses de los interesados y la actitud auto-optimizadora de los actores, representada en la teoría de los juegos, a menudo resulta en comportamientos de las partes interesadas no cooperativas incluso cuando el comportamiento cooperativo es más beneficioso para todas las partes (Madan, 2010). Otra ventaja de la teoría de los juegos sobre los métodos tradicionales de simulación y optimización cuantitativa es su capacidad para simular diferentes aspectos del conflicto, incorporar varias características del problema y predecir las posibles resoluciones en ausencia de información cuantitativa, es decir, puede ayudar a resolver el conflicto basado en el conocimiento cualitativo de las ganancias de los jugadores; esto permite manejar los aspectos socioeconómicos de los conflictos y la planificación, el diseño y el problema político cuando la información cuantitativa no está fácilmente disponible (Madan, 2010). Además, la teoría de juegos puede ayudar a proporcionar algunas ideas de planificación, política y diseño que no estarían disponibles en otros métodos de ingeniería de sistemas tradicionales (Madan, 2010). Por su parte, Bagwell y Wolinsky (2002) establecieron que la teoría de los juegos se ha convertido en el lenguaje estándar de la organización industrial; ya que, la teoría de la organización industrial se presenta ahora casi exclusivamente en términos de modelos teóricos de juegos, pero la relación no es totalmente unilateral, por lo que tenemos en primer lugar: (a) que las necesidades 
de la organización industrial retrocedieron y ejercieron una influencia general en la agenda de la teoría de los juegos; (b) en segundo lugar, las ideas que surgieron de los problemas en la organización industrial ganó importancia independiente como temas de juego teórico en su propio derecho; y (c) en tercer lugar, es sobre todo a través de la organización industrial que la teoría del juego fue llevada a la escala de las economías y alcanzó su posición actual como una rama fundamental de la teoría económica. Por todos estos motivos se considera de mucha importancia el análisis de las aplicaciones de la teoría de juegos en los procesos de dirección y administración estratégica de las empresas, ya que todas las empresas están inmersas en un sistema integrado que cuenta con recursos limitados y las acciones de una empresa afectan las decisiones de otras empresas, decisiones que por lo general son estratégicas y en ocasiones, de aquellas decisiones depende la vida misma de las empresas.

\section{Marco Teórico y Conceptual}

Los términos que a lo largo del artículo se nombrarán se definen a continuación:

\section{Gestión estratégica}

La gestión estratégica debe ser vista desde dos perspectivas que son el ámbito privado y el público, ya que son dos sectores con diferentes políticas y procedimientos, como nos plantean Ring y Perry (1985) que establecen que los gestores estratégicos de los sectores público y privado operan en contextos diferentes que generan restricciones específicas en sus comportamientos y elecciones; por lo que la aplicación de modelos de gestión estratégica del sector privado al sector público es problemática; y que se necesitan modelos generales de gestión estratégica. Indican además que el contexto de la gestión estratégica puede definirse como el rol social de la organización, y el medio ambiente, el tecnológico y el humano; estos contextos y limitaciones pueden implicar que los criterios de evaluación de la gestión pública difieran notablemente de los de la gestión del sector privado. Para Ring y Perry (1985) existen distinciones básicas entre los sectores público y privado, y son fundamentales para entender las diferencias en los procesos de gestión estratégica; quizás la más fundamental de estas diferencias deriva de la ley orgánica: las constituciones; ya que en un esfuerzo por mantener una separación de poder, los redactores constitucionales dividieron a los formuladores de políticas de los ejecutores de políticas; además, en la mayoría de las organizaciones públicas existe autonomía de los empleados y tienen una delicada misión social; por el contrario, las organizaciones del sector privado operan generalmente en el marco de un número limitado de objetivos relativamente estables como el crecimiento, la rentabilidad o la cuota de mercado.

Por su parte, Moore (1995) establece que los elementos centrales de una buena gestión estratégica que tienen un impacto en la efectividad estratégica son: (a) el contexto organizacional (interno y externo); (b) el contenido de la estrategia (incluida la información, la construcción de una lógica y la anticipación de los mecanismos de activación); (c) el proceso de estrategia (incluyendo las formas en que se organiza la información, qué razones se comunican y qué patrones de actividad y niveles de compromiso se producen a lo largo); y (d) el proceso de iteración o regreso a etapas anteriores en el desarrollo de una estrategia. Con relación a esto, Bao (2015) establece que la gestión estratégica está relacionada con la supervivencia y el crecimiento de las organizaciones e incluye las siguientes dimensiones: (a) el "efecto global" o el "efecto global del espacio", que se refiere a la supervivencia y / u organización de las organizaciones en todo el mundo; y (b) los "efectos futuros", que se refieren a la supervivencia en el transcurso del tiempo. 
Además Bao (2015) sugiere que las cuestiones relativas a las partes de las organizaciones que sólo afectan a su supervivencia y / o crecimiento se engloban en los tópicos estratégicos.

Poister (2010) establece que la planificación estratégica adopta un enfoque de "imagen general" que combina el pensamiento futurista, el análisis objetivo y la evaluación subjetiva de valores, metas y prioridades para trazar una dirección y cursos de acción futuros para asegurar la vitalidad, la eficacia y la capacidad de una organización para agregar valor público. Además Poister (2010) argumenta que para que la estrategia sea más significativa en el futuro, se requerirá pasar de la planificación estratégica al proceso más amplio de gestión estratégica, que implica gestionar la agenda estratégica general de una agencia de manera permanente y no episódica, implementándola efectivamente, y para complementar este paso hacia una gestión estratégica más holística, debemos cambiar el énfasis del movimiento de desempeño de una preocupación principal por la medición al proceso más amplio de gestión del desempeño durante la próxima década para concentrarnos más proactivamente en el logro de metas y objetivos estratégicos. Además establece que si bien se ha vuelto omnipresente en el sector público en los últimos 25 años, la planificación estratégica tendrá que desempeñar un papel más crítico en 2020 que en la actualidad; que los gestores públicos deben anticiparse y gestionar el cambio de manera hábil y eficaz frente a nuevas cuestiones que probablemente surjan con una rapidez creciente. Lo que el autor nos sugiere es que se debe implementar la gestión estratégica como un todo analizando a la organización como un sistema dinámico, integrado y en armonía con su entorno. Con relación a esto, Tan y Ding (2015) establecen que la globalización no sólo afecta a los elementos económicos sino también al conocimiento, e incluso a la cultura y al valor; con la creciente cooperación internacional, una nueva teoría de gestión estratégica debe ser suministrada y perfeccionada. Además para Tan y Ding (2015) las teorías básicas de la gestión estratégica, promueven la investigación de gestión estratégica hacia dos dictados: cambio estático a dinámico que es el requisito del cambio competitivo del mercado y de la organización estable a la diversificación, incluso para ampliar la organización de la red, el resultado de la respuesta de la organización al cambio ambiental. Por su parte, Abreu Pederzini y Abreu Pederzini (2016) establecen que los enfoques de la gestión estratégica difieren en las metodologías que valoran, en los enfoques que toman y en las teorías que promueven; y por otra parte, la complicada relación implícita entre la gestión estratégica y la ciencia, que busca sustituir a la cultura clásica de la gestión estratégica, una cultura de la complejidad, también inspirada parcialmente en la ciencia, parece estar desarrollando una gestión estratégica; la complejidad parece estar apareciendo como una alternativa que permite a la dirección estratégica resolver algunos de sus dilemas actuales y, cambiar su relación implícita con la ciencia.

\section{Teoría de juegos}

Para comprender mejor la teoría de juegos se debe definir los siguientes términos, según Turocy y von Stengel (2001):

\section{Jugador}

Un jugador es un agente que toma decisiones en un juego.

Juego

Un juego es una descripción formal de una situación estratégica. 


\section{Juego de suma cero}

Se dice que un juego es de suma cero si para cualquier resultado, la suma de las ganancias para todos los jugadores es cero. En un juego de suma cero de dos jugadores, la ganancia de un jugador es la pérdida del otro jugador, por lo que sus intereses son diametralmente opuestos.

\section{Amplio juego}

Un juego extenso (o juego de forma extensa) describe con un árbol cómo se juega un juego. Representa el orden en que los jugadores hacen movimientos, y la información que cada jugador tiene en cada punto de decisión.

\section{Estrategia}

En un juego en forma estratégica, una estrategia es una de las posibles acciones posibles de un jugador. En un juego extenso, una estrategia es un plan completo de opciones, una para cada punto de decisión del jugador.

\section{Estrategia mixta}

Una estrategia mixta es una aleatorización activa, con probabilidades dadas, que determina la decisión del jugador. Como un caso especial, una estrategia mixta puede ser la elección determinista de una de las estrategias puras dadas.

\section{Forma estratégica}

Un juego en forma estratégica, también llamada forma normal, es una representación compacta de un juego en el que los jugadores al mismo tiempo elegir sus estrategias. Los resultados resultantes se presentan en una tabla con una celda para cada combinación de estrategias.

\section{Estrategia dominante}

Una estrategia domina otra estrategia de un jugador si siempre da una mejor recompensa a ese jugador, independientemente de lo que los otros jugadores están haciendo. Dominan débilmente la otra estrategia si es siempre por lo menos tan buena.

\section{Equilibrio de Nash}

Un equilibrio de Nash, también llamado equilibrio estratégico, es una lista de estrategias, una para cada jugador, que tiene la propiedad de que ningún jugador puede cambiar unilateralmente su estrategia y obtener una mejor recompensa.

\section{Recompensa}

Una recompensa es un número, también llamado utilidad, que refleja la conveniencia de un resultado para un jugador, por cualquier razón. Cuando el resultado es aleatorio, los pagos suelen ponderarse con sus probabilidades. La ganancia esperada incorpora la actitud del jugador hacia el riesgo.

\section{Información perfecta}

Un juego tiene información perfecta cuando en cualquier punto en el tiempo sólo un jugador hace un movimiento, y conoce todas las acciones que se han hecho hasta entonces. 


\section{Racionalidad}

Se dice que un jugador es racional si busca jugar de una manera que maximice su propio beneficio. Se asume a menudo que la racionalidad de todos los jugadores es conocimiento común.

\section{Conocimiento común}

Un hecho es de conocimiento común si todos los jugadores lo saben, y saben que todos lo saben, y así sucesivamente. La estructura del juego se asume a menudo como de conocimiento común entre los jugadores.

\section{¿Qué es la teoría de juegos?}

La teoría de juegos es el estudio formal del conflicto y la cooperación; en donde los conceptos teóricos del juego se aplican cuando las acciones de varios agentes son interdependientes y estos agentes pueden ser individuos, grupos, empresas o cualquier combinación de estos (Turocy \& von Stengel, 2001). Normalmente, se asume una cooperación perfecta entre los tomadores de decisiones para alcanzar las soluciones óptimas del sistema y se supone que estos tomadores de decisiones contribuyen a optimizar la función objetivo sin dar prioridad a sus propios objetivos; sin embargo, en la teoría de los juegos cada decisor juega el juego para optimizar su propio objetivo, sabiendo que las decisiones de otros jugadores afectan su valor objetivo y que su decisión afecta a los pagos de los demás y las decisiones (Madan, 2010). Los juegos son objetos matemáticos definidos, que consisten en un conjunto de jugadores, un conjunto de estrategias (opciones o movimientos) a su disposición y especificación de las ganancias de los jugadores para cada combinación de dichas estrategias (posibles resultados del juego) (Madan, 2010). Por su parte, Osborne (2004) establece que la teoría del juego consiste en una colección de modelos y un modelo es una abstracción que usamos para entender nuestras observaciones y experiencias; esto implica que percibimos las relaciones entre las situaciones, aislando los principios que se aplican a una serie de problemas, para que podamos encajar en nuestro pensamiento nuevas situaciones que nos encontramos. Además para Osborne (2004) el modelado de la teoría del juego comienza con una idea relacionada con algún aspecto de la interacción de los tomadores de decisiones; expresamos esta idea precisamente en un modelo, incorporando rasgos de la situación que parecen ser relevantes. Con relación a esto, para Ogot (2011) la teoría de juegos ofrece una excitante vía para analizar y desarrollar modelos de toma de decisiones aplicables a la gestión estratégica; los modelos pueden derivarse desde una perspectiva teórica, desarrollada a partir de datos empíricos, o una combinación de ambos.

\section{Teoría del Juego en la Gestión Estratégica}

La primera contribución de la teoría de juegos a la organización industrial fue la introducción de un lenguaje, en donde los modelos se describen de una manera precisa y económica utilizando formatos familiares estándar, y los conceptos básicos de solución no cooperativa son comúnmente empleados; y una clara ventaja de esta estandarización es la mejora de la accesibilidad, ya que un teórico político formal o un matemático pueden acceder con relativa facilidad a los escritos de la teoría de la organización industrial moderna, sin una larga introducción a la cultura específica del mundo (Bagwell \& Wolinsky, 2002). Con relación a esto, Ogot (2011) estableció que la teoría de juegos proporciona un lenguaje formal para describir procesos de toma de decisiones conscientes y orientadas a objetivos que involucran a uno o más jugadores, donde hay una interdependencia de los resultados. Otro aspecto a destacar es que la 
teoría de los juegos proporciona un marco analítico general para modelar las decisiones económicas interrelacionadas, motivo por el cual los investigadores aplicados han utilizado esta teoría para probar varios modelos diferentes de comportamiento estratégico; los economistas de la organización industrial, por ejemplo, han modelado el alcance de la competencia oligopolística usando modelos de teoría de juego de competencia de precios y cantidades (Bresnahan \& Reiss, 1991). Con relación a esto, Ginevičius y Krivka (2008) establecieron que entre los modelos de estructura de mercado clásicos (competencia pura, monopolio, oligopolio y competencia monopolística), los modelos de oligopolio son los que más atraen la atención de los científicos en los últimos años; por un lado, debido a la difusión e importancia de los mercados de oligopolio en la economía moderna (por ejemplo, los mercados de productos alimenticios, el mercado de las drogas, el mercado de la cerveza y el alcohol ligero, el mercado de los servicios de telefonía celular y algunos otros pueden ser considerados oligopolios en Lituania). Además para Ginevičius y Krivka (2008) el análisis de los mercados de oligopolios es probablemente el más difícil de todas las estructuras del mercado, por lo que la importancia de la interacción de los participantes en el mercado es el principal factor que distingue el mercado de oligopolios de otras estructuras de mercado de competencia no perfectas, ya que las acciones de cualquier actor del mercado (la elección de precios y cantidades a producir) afectan directamente al equilibrio del mercado e indirectamente a todos los competidores. Todo esto nos lleva a sugerir que la teoría de juegos se puede aplicar y se aplica en todos los casos en donde existe una guerra de precios es decir una escalada que es perjudicial para todos los competidores pero que es una de las estrategias más aplicadas en los mercados competitivos. Con relación a esto, Ogot (2011) establece que muchas de las decisiones de estrategia empresarial implican resultados interdependientes y por lo tanto parecen prestarse a la teoría de los juegos. Además, Ogot (2011) sugiere que la fortaleza de la teoría de los juegos en la gestión estratégica reside en su capacidad para proporcionar conocimientos sobre ambientes y estrategias competitivas.

Para Lahkar (2012) también ha habido una investigación activa en la aplicación de la teoría del juego evolutivo a determinados problemas económicos y sociales; algunas de estas aplicaciones han sido en las áreas de precios de externalidad, congestión en carreteras y redes informáticas, surgimiento de segregación residencial y dispersión de precios. Además, Lahkar (2012) establece que la teoría evolutiva de los juegos ha mejorado considerablemente nuestra comprensión de los fundamentos dinámicos del comportamiento de equilibrio en la sociedad; basándose en normas más realistas y racionalmente racionales del comportamiento humano, nos permite una mejor apreciación de las condiciones bajo las cuales podemos esperar que el equilibrio se funda; al mismo tiempo, este enfoque también puede explicar la persistencia del comportamiento de desequilibrio en ciertos fenómenos económicos o sociales. Con relación a esto, Osborne (2004) sugiere que el razonamiento teórico del juego impregna la teoría económica y se utiliza extensamente en otras ciencias sociales y del comportamiento.

\section{Discusión y Conclusiones}

La aplicabilidad de la teoría de juegos en la gestión estratégica es muy amplia, pero se debe de tener presente el ámbito en donde se desarrollará la gestión, ya que existen diferencias entre el ámbito público y el privado. Si nos enfocamos en las empresas, el actual nivel de competitividad de los mercados provocan guerras de precios en los que las empresas se ven involucradas y esta guerra de precios tiene como consecuencias pérdidas para todas las empresas involucradas, pero la reducción de precios es una estrategia que dependiendo del mercado y 
como se la aplique puede generar ganancias en algunos competidores. La necesidad de generar ganancias en los mercados competitivos actuales hace necesaria la aplicación de la teoría de juegos ya que su fundamento matemático hace que las decisiones que se tomen sean las más adecuadas.

\section{Bibliografía}

Abreu Pederzini, G. D., \& Abreu Pederzini, G. D. (2016). Strategic management cultures: historical connections with science. Journal of Management History, 22(2), 214-235.

Bagwell, K., \& Wolinsky, A. (2002). Game theory and industrial organization. Handbook of Game Theory with Economic Applications, 3, 1851-1895.

Bao, G. (2015). What theories are needed for strategic management?. Nankai Business Review International, 6(4), 433-454.

Bresnahan, T. F., \& Reiss, P. C. (1991). Empirical models of discrete games. Journal of Econometrics, 48(1), 57-81.

Ginevičius, R., \& Krivka, A. (2008). Application of game theory for duopoly market analysis. Journal of Business Economics and Management, (3), 207-217.

Lahkar, R. (2012). Evolutionary game theory: an exposition. Indian Growth and Development Review, 5(2), 203-213.

Madani, K. (2010). Game theory and water resources. Journal of Hydrology, 381(3), 225-238.

Moore, S. (1995). Making sense of strategic management: towards a constructive guide. Management Decision, 33(1), 19-23.

Ogot, M. (2011). Game Theory in Strategic Management. Unpublished Journal.

Osborne, M. J. (2004). An introduction to game theory (Vol. 3, No. 3). New York: Oxford University Press.

Poister, T. H. (2010). The future of strategic planning in the public sector: Linking strategic management and performance. Public Administration Review, 70(s1), s246-s254.

Roth, A. E. (2002). The economist as engineer: Game theory, experimentation, and computation as tools for design economics. Econometrica, 70(4), 1341-1378.

Tan, L., \& Ding, J. (2015). The frontier and evolution of the strategic management theory: A scientometric analysis of Strategic Management Journal, 2001-2012. Nankai Business Review International, 6(1), 20-41.

Turocy, T. L., \& von Stengel, B. (2001). Game Theory*: Draft prepared for the Enciclopedia of Information Systems. Dept. Math., London School Econ., London, UK, Tech. Rep. LSECDAM-2001-09. 